\title{
A Strategy-Proof Radio Spectrum Auction Mechanism in Noncooperative Wireless Networks
}

\author{
Fan Wu, Member, IEEE, and Nitin Vaidya, Fellow, IEEE
}

\begin{abstract}
With the growing deployment of wireless communication technologies, radio spectrum is becoming a scarce resource. Thus, mechanisms to efficiently allocate the available spectrum are of interest. In this paper, we model the radio spectrum allocation problem as a sealed-bid reserve auction, and propose SMALL, which is a Strategy-proof Mechanism for radio spectrum ALLocation. Furthermore, we extend SMALL to adapt to multiradio spectrum buyers, which can bid for more than one radio. We evaluate SMALL with simulations. Simulation results show that SMALL has good performance in median to large scale spectrum auctions.
\end{abstract}

Index Terms-Wireless network, channel allocation, security, mechanism design, auction

\section{INTRODUCTION}

$\mathrm{R}$ ADIO spectrum is becoming a scarce resource due to the increasing deployment of wireless communication technologies. For historical reasons, much of the radio spectrum is statically allocated. The inefficiency of such an allocation is twofold. On one hand, the static allocation does not consider spatial and temporal variation of the spectrum. Large chunks of radio spectrum are left idle most of the time at a lot of places. On the other hand, many new wireless applications cannot find enough radio spectrum to operate on. Therefore, redistribution of idle radio spectrum is important to make a better utilization of the radio spectrum.

To redistribute radio spectrum, a natural way is to use auction, which is a process of buying and selling goods by offering them up for bid, taking bids, and then selling the item(s) to the highest bidder(s). Since 1994, the Federal Communications Commission (FCC) has conducted auctions of licenses for radio spectrum [7]. While FCC auctions target only large wireless applications, we consider small wireless application buyers, such as community wireless networks and home wireless networks. These small buyers can search for and reuse idle chunks of radio spectrum.

However, designing a practical spectrum auction mechanism has its own challenges. One of the major challenges is spatial reusability of the radio spectrum, which differentiate it from conventional goods. Spectrum

- F. Wu is with the Department of Computer Science and Engineering, Shanghai Key Laboratory of Scalable Computing and Systems, Shanghai Jiao Tong University, 3-537 SEIEE Building, 800 Dongchuan Road, Shanghai 200240, China. E-mail: fwu@cs.sjtu.edu.cn.

- N. Vaidya is with the Department of Electrical and Computer Engineering and the Coordinated Science Lab, University of Illinois at UrbanaChampaign, 458 Coordinated Science Laboratory, MC-228, 1308 West Main Street, Urbana, IL 61801. E-mail: nhv@illinois.edu.

Manuscript received 13 May 2011; revised 27 Oct. 2011; accepted 14 Feb. 2012; published online 28 Feb. 2012.

For information on obtaining reprints of this article, please send e-mail to: tmc@computer.org, and reference IEEECS Log Number TMC-2011-05-0254. Digital Object Identifier no. 10.1109/TMC.2012.55. buyers, who are within the interference range of each other, cannot use the same spectrum band simultaneously, while well-separated buyers can. Furthermore, the problem of finding the optimal spectrum allocation is NP-complete [5], [31]. Another major challenge, which is not limited only to spectrum auctions but applies to traditional auctions in general, is strategy-proofness (see Section 2.2 for the definition), which intuitively means that reporting true valuation as a bid maximizes one's payoff. Since the participants are rational and always want to maximize their own objectives, it is likely that the participants would strategically manipulate the auction, if doing so can benefit themselves. Therefore, truthfully behaving spectrum buyers can be discouraged from participating in the auction, if strategy-proofness is not guaranteed.

Recently, Zhou and Zheng proposed TRUST [36] and VERITAS [35] to support open auction-based spectrum redistribution. Both auction mechanisms achieve strategy proofness. TRUST takes into account both buyers and sellers' valuation on the channels, and elegantly integrates double auction and radio spectrum allocation. TRUST enables spectrum reuse and can improve spectrum utilization. Unfortunately, to guarantee the strategy proofness, TRUST has to sacrifice a good transaction, which includes a channel and a group of buyers. When TRUST is used, not all of the channels can be sold, and the number of sacrificed buyers grows almost linearly with the number of buyers. Furthermore, TRUST does not support the need from a buyer for multiple channels. Unlike TRUST, VERITAS does not sacrifice any good transaction, and provides the support for bidding multiple channels. But VERITAS does not consider seller's valuation of the channels, which may include the leasing expense of the channel. A channel may be sold at a price much lower than the seller's valuation, and thus the incentive of the seller to resell a channel may be hurt.

In this paper, we present a Strategy-proof Mechanism for radio spectrum ALLocation (SMALL). SMALL is a 
sealed-bid reserve auction mechanism, in which all bidders simultaneously submit sealed bids so that no bidder knows the bid of any other participant, and a channel may not be sold if the final bid is not high enough to satisfy the seller. SMALL supports radio spectrum reuse, bidding for multiple channels, and protects channel seller's incentive.

We make the following contributions in this paper:

- First, we model the radio spectrum allocation problem as a sealed-bid reserve auction, and design a novel auction mechanism, called SMALL, for singleradio spectrum auction. We prove that SMALL is a strategy-proof auction mechanism.

- Second, we extend SMALL to support multiradio spectrum auction, and prove that the enhanced SMALL again achieves strategy proofness.

- Finally, we evaluate SMALL using extensive simulations. Our simulation results show that SMALL achieves good performance in terms of buyer satisfaction ratio, channel utilization, and budget efficiency in median to large scale spectrum auctions.

The rest of this paper is organized as follows: In Section 2, we present technical preliminaries. In Section 3, we describe our spectrum auction mechanism-SMALL, and prove its strategy-proofness. In Section 4, we extend SMALL to support multi-radio spectrum auction. In Section 5, we report evaluation results of SMALL. In Section 6, we review related work. In Section 7, we draw conclusions and discuss future work.

\section{Technical Preliminaries}

In this section, we present our game model for the spectrum allocation problem, and review some useful solution concepts from game theory and mechanism design.

\subsection{Game Model}

We consider a static scenario in which there is a large wireless service provider, called "seller," who possesses a number of orthogonal spectrum channels and wants to lease out regionally unused channels; and there is a set of static nodes, called "buyers," such as WiFi access points, who want to lease channels in order to provide services to their users. A channel can be leased to multiple buyers, if these buyers can transmit simultaneously and receive signals with an adequate Signal to Interference and Noise Ratio (SINR). We model this problem as a sealed-bid reserve auction, in which all buyers simultaneously submit sealed bids so that no buyer knows the bid of any other participant, and a channel may not be sold if the final bid is not high enough to satisfy the seller. The objective of the auction is to efficiently allocate the channels to the buyers based on their bids, without violating interference conditions between the buyers.

We assume that the seller is trustworthy, and has a set $C=\left\{c_{1}, c_{2}, \ldots, c_{m}\right\}$ of orthogonal and homogenous channels to lease. Each channel can be simultaneously used by multiple nonconflicting buyers. The seller has a reserve price for each of the channels, denoted by $S=\left\{s_{1}, s_{2}, \ldots, s_{m}\right\}$. A reserve price can be an operating expense, if the seller put a channel on auction. A channel can be leased to one or a group of non-conflicting buyers if the sum of the bids is not lower than the reserve price. (We will define buyer group in Section 3.1.)

We also assume that there is a set $N=\{1,2, \ldots, n\}$ of buyers. Each buyer $i \in N$ only requests a single channel and has a valuation $v_{i}$ on the channel. The channel valuation can be the revenue got by the buyer for serving her subscribers. (In Section 4, we will consider an extended model, in which buyers can be equipped with multiple radios and bid for multiple channels. The channel valuations are identical for multiple radios/virtual buyers, because the buyer can serve more subscribers or provide better service quality, when getting more channels.) The channel valuation $v_{i}$ is a private information to the buyer $i$. It is also known as type in the literature. In the auction, the buyers simultaneously submit their sealed bids, denoted by $\left\{b_{1}, b_{2}, \ldots, b_{n}\right\}$, which are based on their types. The auction mechanism determines the set of winning buyers, channel allocation to the winners, and the charge of each winner. Denote the charge of a buyer $i \in N$ by $p_{i}$. Then, we define the utility $u_{i}$ of buyer $i$ to be the difference between her valuation $v_{i}$ on the channel and the charge $p_{i}$ :

$$
u_{i}=v_{i}-p_{i} .
$$

We assume that the buyers are rational. The objective of each buyer is to maximize her own utility. A buyer has no preference over different outcomes, if the utilities are same to the buyer herself. We also assume that the buyers do not collude with each other.

In contrast to players' individual objective, the overall objective of the auction mechanism is to improve channel utilization and buyer satisfaction ratio. Here, channel utilization is the sum of allocated channels of all the winning buyers; buyer satisfaction ratio is the percentage of winning buyers in the auction. Furthermore, to avoid the buyers paying too high prices, a good auction mechanism should also be budget efficient, which means the overpayment, between buyers' total charge and sellers' total valuation/reserve price, should be small.

\subsection{Solution Concepts}

We review the important solution concepts used in this paper from game theory and mechanism design. First, we recall the definition of Dominant Strategy:

Definition 1 (Dominant strategy [9], [21]). A dominant strategy of a player is one that maximizes her utility regardless of what strategies other players choose. Specifically, $a_{i}$ is player $i$ 's dominant strategy if, for any $a_{i}^{\prime} \neq a_{i}$ and any strategy profile of the other players $a_{-i}$,

$$
u_{i}\left(a_{i}, a_{-i}\right) \geq u_{i}\left(a_{i}^{\prime}, a_{-i}\right) .
$$

Before recalling the definition of Strategy-proof Mechanism, we define direct-revelation mechanism first. A direct-revelation mechanism is a mechanism in which the only actions available to players are to make claims about their preferences to the mechanism. In our channel auction, the strategy of a buyer $i \in N$ is reporting a bid $b_{i}=a_{i}\left(v_{i}\right)$, based on her actual channel valuation $v_{i}$. A direct-revelation mechanism is strategy proof if it satisfies two conditions, 


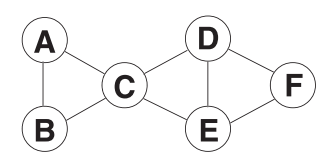

Fig. 1. A toy network with six buyers $(A-F)$.

incentive compatibility and individual rationality. Incentive compatibility means reporting truthful information is a dominant strategy for each player. Individual rationality means each player can always achieve at least as much expected utility from faithful participation as without participation. The formal definition of Strategy-proof Mechanism is as follows:

Definition 2 (Strategy-proof mechanism [17], [25]). A direct-revelation mechanism is strategy proof if revealing truthful information is a dominant-strategy equilibrium.

\section{Strategy-Proof Radio Spectrum ALLOCATION MeChanISM-SMALL}

In this section, we present our design of radio spectrum auction mechanism-SMALL, and prove its strategy proofness.

\subsection{Design of SMALL}

SMALL is composed of three algorithms: buyer grouping, winner selection, and charge determination. Since the seller is a trustworthy authority, we let the seller serves as auctioneer and perform the computation of the three algorithms.

\subsubsection{Buyer Grouping}

Since the channels can be spatially reused, SMALL divides the buyers into multiple nonconflicting groups, each of which can be assigned to a distinguished channel. To prevent the buyers manipulating the auction, the grouping need to be independent of the buyers' bids. Therefore, SMALL first constructs a conflict graph of the buyers. Any pair of buyers, who are in the interference range of each other, have a line connecting them in the conflict graph. Then, buyer groups can be calculated by any existing graph coloring algorithm [29] which is independent of buyers' bids, such that no buyer can be in multiple groups. We note that the buyers cannot determine which group they are in by themselves, when the above grouping strategy is used. We denote the calculated buyer groups by $G=\left\{g_{1}, g_{2}, \ldots, g_{l}\right\}$.

Fig. 1 shows a toy network with six buyers $(A-F)$. There are several grouping results, e.g., $g_{1}=\{A, D\}, g_{2}=$ $\{B, E\}$, and $g_{3}=\{C, F\}$.

\subsubsection{Winner Selection}

We now determine an integrated group bid for each buyer group. A natural way to calculate the group bid is to simply add all the bids from the group members together. However, this way may allow the buyers to manipulate the group bid by reporting untruthful bids. Thus, the strategy proofness of the auction can be hurt. Therefore, to guarantee the strategy proofness, we sacrifice the buyer with the smallest bid in each group, and define an integrated group bid $\sigma_{j}$ for each group $g_{j} \in G$ as:

$$
\sigma_{j}=\left(\left|g_{j}\right|-1\right) \cdot \min \left\{b_{k} \mid k \in g_{j}\right\} .
$$

By this way, the group bid is independent of valid members' bids (i.e., the bids except the smallest one) in each group. Such a definition of group bid is reasonable, because the strategy proofness can be guaranteed by sacrificing the buyer that makes the least contribution in a group. Then, we get a set of group bids $\Sigma=\left\{\sigma_{1}, \sigma_{2}, \ldots, \sigma_{l}\right\}$.

Next, SMALL sorts the channels by reserve price in nondecreasing order and buyer groups by group bid in nonincreasing order:

$$
\begin{aligned}
& C^{\prime}: s_{1}^{\prime} \leq s_{2}^{\prime} \leq \cdots \leq s_{m}^{\prime}, \\
& G^{\prime}: \sigma_{1}^{\prime} \geq \sigma_{2}^{\prime} \geq \cdots \geq \sigma_{l}^{\prime} .
\end{aligned}
$$

Here, each $s_{i}^{\prime}\left(\sigma_{j}^{\prime}\right)$ corresponds to a unique reserve price in $S$ (group bid in $\Sigma$ ). In the case of ties, the ordering is random, with each tied channel/group having an equal probability of being ordered prior to the other one.

Next, SMALL finds the maximal number of trades $k$, s.t.

$$
\sum_{i=1}^{k} s_{i}^{\prime} \leq \sum_{i=1}^{k} \sigma_{i}^{\prime} .
$$

Finally, the winning groups are the first $k$ buyer groups in $G^{\prime}$, and the first $k$ channels in $C^{\prime}$ are leased to each of the corresponding winning groups. In each of the winning groups, the buyers, except the one with the smallest bid in that group, are winning buyers. In the case of ties, i.e., more than one buyers report the smallest bid in the group, each tied buyer has an equal probability of being selected as a winning buyer. ${ }^{1}$

Noting that exactly one buyer must be sacrificed for each channel leased, the total number of sacrificed buyers has an upper bound $m$, which is the number of channels. Since singleton groups cannot compete for channels, as their group bid would be zero, SMALL is more appropriate to be used in a radio spectrum auction with relatively large number of buyers scattered in a large area.

\subsubsection{Charging}

Each winning buyer $i \in g_{j}$ is charged an even share of her group bid, which is also equivalent to the smallest bid in the group:

$$
p_{i}=\frac{\sigma_{j}}{\left|g_{j}\right|-1}=\min \left\{b_{k} \mid k \in g_{j}\right\} .
$$

In each winning group, we exclude the buyer with the smallest bid, and charge the others with the smallest bid, in order to make the charge be independent of winners' bids.

The seller collects all the payments:

$$
q=\sum_{j=1}^{k} \sigma_{j}^{\prime} .
$$

1. We have to note that SMALL is designed to guarantee the truthfulness of channel auction. However, it does not provide any guarantee on the optimality of the channel allocation result. The optimality of the channel allocation result relies on the output of the graph coloring algorithm. We left the problem of selecting the most suitable graph coloring algorithm to our future work. 
We note that the auction is budget balanced, which means that the total amount of the buyers' payments is equal to the total amount of the payments to be received by the seller [17].

Combining (1) and (2), we get

$$
q \geq \sum_{i=1}^{k} s_{i}^{\prime}
$$

Therefore, the seller's profit is guaranteed. We note that we do not specify the algorithm for dividing the seller's revenue to each channel successfully leased. One of the possible ways is to divide the revenue proportionally to the channels' reserve prices.

In the next section, we will prove that buyers' truthfulness is also guaranteed.

\subsection{Strategy Proofness}

Lemma 1. If SMALL is used, reporting the true channel valuation as a bid is a dominant strategy for each buyer.

Proof. We will show that a buyer cannot increase her utility by proposing a bid other than her true valuation. That is to say, truthfulness is a dominant strategy.

Consider a buyer $i$ in group $g_{j}$ with valuation $v_{i}$. Let $b_{\min }=\min \left\{b_{k} \mid k \in g_{j}\right\}$. We distinguish two cases:

1. The buyer $i$ is in a winning group, when bidding true valuation, i.e., $b_{i}=v_{i}$. Her utility is

$$
u_{i}= \begin{cases}v_{i}-b_{\min } & \text { if } b_{i}>b_{\min } \\ 0 & \text { if } b_{i}=b_{\min }\end{cases}
$$

Consider the following two cases:

a. $\quad b_{i}>b_{\min }$ : Buyer $i$ is a winner. Suppose buyer $i$ reports another bid $b_{i}^{\prime} \neq b_{i}$. If she still wins the channel $\left(b_{i}^{\prime} \geq b_{\min }\right)$, then buyer $i^{\prime}$ 's utility is not changed, since the smallest bid is still $b_{\text {min }}$. If she losses the channel $\left(b_{i}^{\prime} \leq b_{\min }\right)$, then buyer $i^{\prime}$ s utility goes to 0 . Therefore, buyer $i^{\prime}$ s new utility $u_{i}^{\prime} \leq u_{i}$.

b. $b_{i}=b_{\min }$ : Suppose buyer $i$ reports a another bid $b_{i}^{\prime} \neq b_{i}$. If she wins a channel $\left(b_{i}^{\prime} \geq \min \left\{b_{k} \mid\right.\right.$ $\left.\left.k \in g_{j} \wedge k \neq i\right\}\right)$ and pay $\min \left\{b_{k} \mid k \in g_{j} \wedge k \neq i\right\}$, then her utility becomes

$$
\begin{aligned}
u_{i}^{\prime} & =v_{i}-\min \left\{b_{k} \mid k \in g_{j} \wedge k \neq i\right\} \\
& \leq v_{i}-b_{\min } \\
& \leq v_{i}-b_{i} \\
& =0 .
\end{aligned}
$$

If she does not win a channel $\left(b_{i}^{\prime} \leq \min \left\{b_{k} \mid k \in\right.\right.$ $\left.\left.g_{j} \wedge k \neq i\right\}\right)$, then her utility is still 0 . Therefore, $u_{i}^{\prime} \leq u_{i}$. Recall that the buyer has no preference over different outcomes, if the utilities are same. Therefore, she has no incentives to report a higher bid and win the channel.

2. The buyer $i$ is not in a winning group, when bidding true valuation, i.e., $b_{i}=v_{i}$. Her utility is

$$
u_{i}=0 .
$$

In this case, the only way to change her utility is to make group $g_{j}$ becomes a winning group, by reporting a higher bid $b_{i}^{\prime}>b_{i}$, when $b_{i}$ is the lowest bid in group $g_{j}$. Suppose group $g_{j}$ becomes a winning group when the buyer $i$ reports $b_{i}^{\prime}>b_{\min }=b_{i}$. If $b_{i}^{\prime}$ is still the smallest bid in group $g_{j}$, she still cannot get a channel and her utility is $u_{i}^{\prime}=0$. If $b_{i}^{\prime} \geq \min \left\{b_{k} \mid k \in g_{j} \wedge k \neq i\right\}$, she may get a channel and pay $\min \left\{b_{k} \mid k \in g_{j} \wedge k \neq i\right\}$. Then, her utility is

$$
\begin{aligned}
u_{i}^{\prime} & =v_{i}-\min \left\{b_{k} \mid k \in g_{j} \wedge k \neq i\right\} \\
& \leq v_{i}-\min \left\{b_{k} \mid k \in g_{j}\right\} \\
& \leq v_{i}-b_{i} \\
& =0 .
\end{aligned}
$$

Therefore, bidding the true valuation is a dominant strategy for each buyer.

From the analysis above, we get that SMALL satisfies incentive compatibility. On one hand, we can see that each truthful buyer's utility is always $\geq 0$. On the other hand, by not taking part in the auction, a buyer cannot get a channel and her utility remains to be 0 . So participating is not worse than staying outside, which satisfies the individual rationality.

Since our mechanism satisfies both incentive compatibility and individual rationality, we have the following theorem:

\section{Theorem 1. SMALL is a strategy-proof mechanism.}

\section{Extension to Multiple Radios}

In the previous section, we considered the scenario in which each buyer only has a single radio. In reality, some access points may be equipped with multiple radios. In this section, we extend our work to adapt to multiple radios having the same communication capabilities. A buyer with multiple radios can provide wireless services on multiple channels. So in the spectrum auction, a multiradio buyer may bid for more than one channels. In the conflict graph, a $r$-radio buyer is represented by at most $r$ virtual buyers inheriting the interference condition of their parent. Since the number of radios $r$ on a buyer may be larger than that of the channels for sale $m$, we require that the number of virtual buyers for a $r$-radio buyer is $\min \{r, m\}$. The virtual buyers also have interference between each other. We assume that buyers have no preference over channels and they do not cheat about the number of radios. Considering that the buyer can serve more subscribers or provide better service quality, when getting more channels, we also assume that the channel valuations are identical for the virtual buyers. Hence, we let the virtual buyers share the same channel valuation and bid from her parent. The parent buyer's utility is the sum of the utilities got by her virtual child buyers. Since a buyer can have multiple virtual child buyers and report multiple bids, the previous auction mechanism cannot be directly applied here. In this section, first, we show an example, in which a multiradio buyer can benefit by misreporting her bids. Then, we present our 


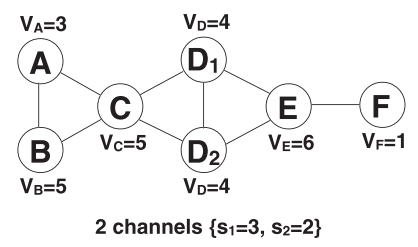

Fig. 2. Buyer $D$ can get a higher utility by bidding $b_{D}^{\prime}=1.5$, when using SMALL.

enhanced SMALL to prevent misreporting when nodes have multiple radios.

\subsection{Example: Multiradio Buyer Can Benefit by Misreporting}

Fig. 2 shows a scenario, in which there are two channels and six buyers $(A-F)$. The reserve prices of the channels are $s_{1}=3$ and $s_{2}=2$. The channel valuations are shown near the buyers. A line between two buyers indicates that they interfere with each other and cannot share the same channel. Among the buyers, $D$ has two radios. Since buyer $D$ has two radios, we duplicate $D$ as $D_{1}$ and $D_{2}$, and connect them with a line. $D_{1}$ and $D_{2}$ inherit the interference condition from $D$.

Suppose the buyers are divided into three nonconflicting groups: $g_{1}=\left\{A, D_{1}, F\right\}, g_{2}=\left\{B, D_{2}\right\}$, and $g_{3}=\{C, E\}$. If the buyers bid their true valuations, then the group bids are $\sigma_{1}=2, \sigma_{2}=4$, and $\sigma_{3}=5$. So the winning groups are $g_{2}$ and $g_{3}$, and the winning buyers are $B$ and $E$. The utilities of $B$ and $E$ are

$$
u_{B}=v_{B}-p_{B}=5-4=1
$$

and

$$
u_{E}=v_{E}-p_{E}=6-5=1,
$$

respectively, while the utilities of $A, C, D$, and $F$ are 0 .

But, buyer $D$ can get a higher utility by unilaterally reporting a bid other than her true valuation. In particular, if $D$ reports $b_{D}^{\prime}=1.5$, then the group bid of $g_{2}$ becomes $\sigma_{2}^{\prime}=1.5$, while the other two remain unchanged. Consequently, the winning groups becomes $g_{1}$ and $g_{3}$, and the winning buyers are $A, D_{1}$, and $E$. The utilities of the winners are

$$
\begin{aligned}
& u_{A}=v_{A}-p_{A}=3-1=2, \\
& u_{D}=u_{D_{1}}+u_{D_{2}}=v_{D}-p_{D_{1}}=4-1=3, \\
& u_{E}=v_{E}-p_{E}=6-5=1 .
\end{aligned}
$$

We can see that $D$ gets a higher utility by misreporting her channel valuation.

Therefore, the previous auction mechanism cannot be directly used when buyers have multiple radios.

\subsection{Design of Enhanced SMALL}

From the previous example, we can observe that a buyer may benefit by manipulating the relative order of the groups, in which the buyer has a virtual child buyer, and holding the smallest bid in one of the group. In particular, the buyer $D$ holds the smallest bid in group $g_{2}$, and changes the relative order of the group bids of buyer groups ( $g_{1}$ and $g_{2}$ ) from $\sigma_{2} \geq \sigma_{1}$ to $\sigma_{1} \geq \sigma_{2}$, by manipulating her bid. Since spectrum resource becomes relatively more scarce compared with the increased number of radios, we assume that all the channels can be sold in the multiradio channel auction.

Instead of sorting the buyer groups by a predefined group bid, the enhanced SMALL sorts the buyer groups by the group size in nonincreasing order:

$$
G^{\prime}:\left|g_{1}^{\prime}\right| \geq\left|g_{2}^{\prime}\right| \geq \cdots \geq\left|g_{l}^{\prime}\right|
$$

while still sorting the channels by reserve price in nondecreasing order:

$$
C^{\prime}: s_{1}^{\prime} \leq s_{2}^{\prime} \leq \cdots \leq s_{m}^{\prime}
$$

In the case of ties, the ordering is random, with each tied channel/group having an equal probability of being ordered prior to the other one.

Same as before, the enhanced SMALL finds the maximal number of trades $k$, s.t.

$$
\sum_{i=1}^{k} s_{i}^{\prime} \leq \sum_{i=1}^{k} g_{i}^{\prime} .
$$

Since it is assumed that all the channels can be sold in the multiradio channel auction, here $k=m$.

The winning groups are $g_{1}^{\prime}, g_{2}^{\prime}, \ldots, g_{k}^{\prime}$, and each winning group can get a channel. The winners in each of the winning groups are the ones except the smallest bidder in that group. The previous tie breaking rule also applies here. The charge to a winner $i \in g_{j}^{\prime}$ is the smallest bid in group $g_{j}^{\prime}$ :

$$
p_{i}=\min \left\{b_{k} \mid k \in g_{j}^{\prime}\right\} .
$$

\subsection{Analysis on SMALL with Multiradio Buyers}

In this section, we prove that the enhanced SMALL is also a strategy-proof mechanism. We prove the following lemma first.

Lemma 2. If the enhanced SMALL is used, reporting the true channel valuation is a dominant strategy for each buyer despite multiple radios.

Proof. Same as before, we will show that a buyer cannot increase her utility by proposing a bid other than her true valuation. In other words, truthfulness is a dominant strategy.

Let's consider a buyer $i$ who has $\hat{r}$ virtual child buyers in the channel auction. Suppose the buyer $i$ has $\hat{t}$ virtual child buyers in the winning groups. Without lose of generality, we assume that virtual buyers $\left\{i_{1}, i_{2}, \ldots, i_{\hat{t}}\right\}$ are in winning groups, while virtual buyers $\left\{i_{\hat{t}+1}, i_{\hat{t}+2}, \ldots, i_{\hat{r}}\right\}$ are in losing groups. Let $\hat{g}\left(i_{k}\right), k \in\{1,2, \ldots, \hat{r}\}$ be the group, to which the virtual buyer $i_{k}$ belongs. Let $u_{i}$ denote buyer $i$ 's utility when she proposes her true valuation as the bid. We distinguish two cases:

1. The buyer $i$ proposes a bid $b_{i}$ higher than her true valuation $v_{i}$, i.e., $b_{i}>v_{i}$. For each virtual buyer $i_{k}$, if she becomes a winner, while she would not if bidding truthfully, then $i_{k}$ must belong to $\left\{i_{1}, i_{2}, \ldots, i_{\hat{r}}\right\}$ and the utility on virtual buyer $i_{k}$ becomes 
$u_{i_{k}}^{\prime}=v_{i}-\min \left\{b_{x} \mid x \in \hat{g}\left(i_{k}\right) \wedge x \neq i_{k}\right\} \leq 0=u_{i_{k}}$.

Otherwise, $u_{i_{k}}^{\prime}=u_{i_{k}}$. Therefore, buyer $i^{\prime}$ s new utility

$$
u_{i}^{\prime}=\sum_{k=1}^{\hat{r}} u_{i_{k}}^{\prime} \leq \sum_{k=1}^{\hat{r}} u_{i_{k}}=u_{i} .
$$

2. The buyer $i$ proposes a bid $b_{i}$ lower than her true valuation $v_{i}$, i.e., $b_{i}<v_{i}$. We first consider a virtual buyer $i_{k} \in\left\{i_{1}, i_{2}, \ldots, i_{\hat{t}}\right\}$. If $b_{i}$ becomes or still be the smallest bid in group $\hat{g}\left(i_{k}\right)$, virtual buyer $i_{k}$ cannot get a channel and her utility is $u_{i_{k}}^{\prime}=0 \leq u_{i_{k}}$. Otherwise, $u_{i_{k}}^{\prime}=u_{i_{k}}$. We then consider a virtual buyer $i_{k^{\prime}} \in\left\{i_{\hat{t}+1}, i_{\hat{t}+2}, \ldots, i_{\hat{r}}\right\}$. Since the group order in $G^{\prime}$ does not depend on the bids, neither $\hat{g}\left(i_{k}\right)$ can become a winning group, nor $i_{k^{\prime}}$ can become a winner. Consequently, $u_{i_{k^{\prime}}}^{\prime}=0$. Therefore, buyer $i^{\prime}$ s new utility $u_{i}^{\prime} \leq u_{i}$.

Therefore, bidding the true valuation is a dominant strategy for each buyer.

Then, we get the following theorem:

Theorem 2. The enhanced SMALL is a strategy-proof mechanism despite multiple radios.

Proof. On one hand, if a buyer bids truthfully, each of her radio (virtual buyer) can get a nonnegative utility, because the virtual buyer's bid is always not lower than her charge (i.e., the smallest bid in the virtual buyer's group). Consequently, the truthful buyer's utility is always $\geq 0$. On the other hand, by not taking part in the auction, a buyer cannot get a channel and her utility remains to be 0 . So participating is not worse than staying outside, which satisfies the individual rationality.

From Lemma 2, we get that the enhanced SMALL satisfies incentive compatibility despite multiple radios. Since our mechanism satisfies both incentive compatibility and individual rationality, we conclude that the enhanced SMALL is a strategy-proof mechanism despite multiple radios.

\section{Numerical Results}

We implement SMALL and evaluate its performance using network simulations. The objective of our simulations is twofold. One is to measure the influence of our mechanisms on the system performance. The other one is to evaluate the impact of our design on budget efficiency, which can be measure by the difference between node's total payment and sellers' total valuation/reserve price.

\subsection{Methodology}

We implement SMALL based on a greedy graph coloring algorithm [28], and compare its performance with VERITAS. Buyers are randomly distributed in the terrain area of $2,000 \times 2,000$ meters by default. The number of buyers varies from 20 to 400 . The radio interference range of each node is set to 425 meters. The numbers of channels for leasing can be 5,10 , or 15 . In the single-radio simulation, each buyer only has a single radio and reports one bid; while in the multiradio simulation, each buyer is equipped with three radios and can bid for up to three channels. We assume that buyers' channel valuations are randomly distributed over $(0,1]$, and seller's reserve prices are randomly distributed over $(0,2] .^{2}$ All the results on performance are averaged over 200 runs.

Metrics: We evaluate two metrics:

- Buyer satisfaction ratio: Buyer satisfaction ratio is the percentage of buyers who get at least one channel in the auction.

- Channel utilization: Average number of radios allocated to each channel. Buyer satisfaction ratio and channel utilization reflect the performance of a channel auction mechanism.

- Overpayment: Overpayment is the difference between buyers' total payment and sellers' total valuation/reserve price. This metric reflects the budget efficiency of a channel auction.

Our simulations are to compare the performance of SMALL with existing strategy-proof channel auction mechanisms, such as VERITAS and TRUST. Since TRUST is a strategy-proof double auction, which also considers the truthfulness of the sellers, we only compare the buyer satisfaction ratio and channel utilization of SMALL with that of VERITAS. Since VERITAS does not consider seller's valuation/reserve price on channels, we compare the overpayments of SMALL with TRUST. Our simulation results show that SMALL achieves improved performance compared with VERITAS in many cases. In particular, compared with VERITAS, SMALL can achieve better buyer satisfaction ratio and channel utilization, except for small scale spectrum auctions, in which the network has only a small number of buyers, the number of channels is small, or the terrain area is small. Therefore, while VERITAS is a good choice for small scale spectrum auctions, SMALL is more suitable for median to large scale spectrum auctions. Simulation results also show that SMALL always provides smaller overpayment than TRUST.

\subsection{Buyer Satisfaction Ratio}

We present the simulation results on buyer satisfaction ratio in this section.

Figs. $3 \mathrm{a}$ and $3 \mathrm{~b}$ show buyer satisfaction ratios of SMALL and VERITAS for auctioning 15 channels. Two sets of results are presented. In one set, each buyer only has a single radio. In the other set, each buyer is equipped with three radios. From the figure, we can see that VERITAS performs better than SMALL when the number of (virtual) buyers is no larger than 120 (In the case of single radio, the threshold is 120 buyers; In the case of three radios, the threshold is 40 buyers, which is equal to 120 virtual buyers.). This is because VERITAS does not need to sacrifice any bid to guarantee the strategy proofness. However, when the number of (virtual) buyers is larger than 120, the buyer satisfaction ratio achieved by SMALL becomes higher

2. The ranges of buyers' channel valuations and seller's reserve prices can be different from the ones used here. However, the evaluation results of using different ranges are identical. Therefore, we only show the results for the above ranges in this paper. 


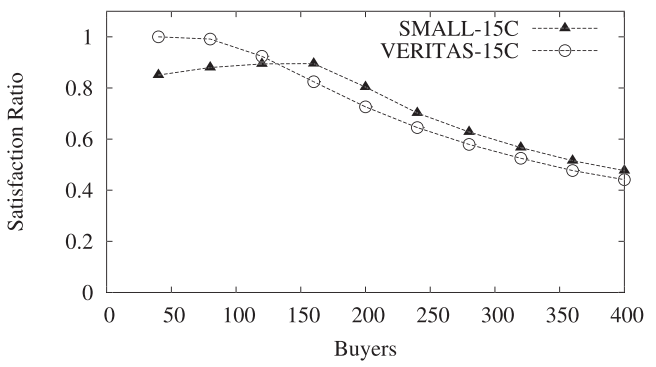

(a) Single radio

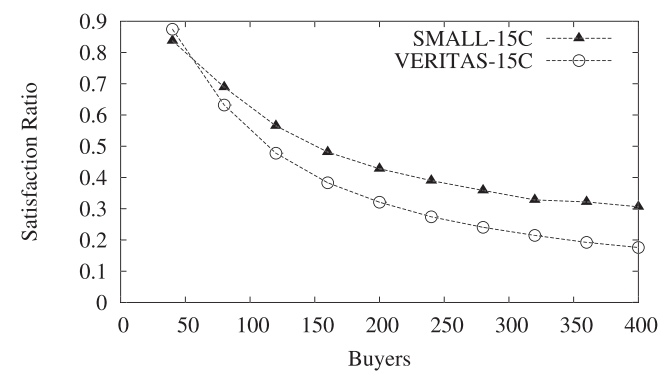

(b) 3 radios

Fig. 3. Buyer satisfaction ratios of SMALL and VERITAS for auctioning 15 channels.

than that of VERITAS. The reason for this is that VERITAS's greedy channel allocation algorithm is lack of consideration of the whole network. SMALL provides better buyer satisfaction ratio in networks with relatively large number of buyers.

Fig. 4 shows buyer satisfaction ratios of SMALL and VERITAS for auctioning 5, 10, and 15 channels, among 200 buyers, when every buyer has a single radio and three radios, respectively. The satisfaction ratio of SMALL is higher than that of VERITAS, only except when every buyer has a single radio and there are five channels for sale.

Fig. 5 shows the buyer satisfaction ratios of SMALL and VERITAS, when there are 200 buyers and 10 channels for auctioning. We can see that SMALL performs worse than VERITAS when the terrain area is $500 \times 500$ meters. This is because the opportunity of channel reuse is very few when the terrain area is small. When the terrain area is too small, the number of buyer groups, whose sizes are larger than 1, is also very small, which results in that SMALL does not have enough buyer groups. However, when the terrain area is relatively large $(1,000 \times 1,000,1,500 \times 1,500$, and $2,000 \times 2,000$ meters), SMALL achieves similar buyer

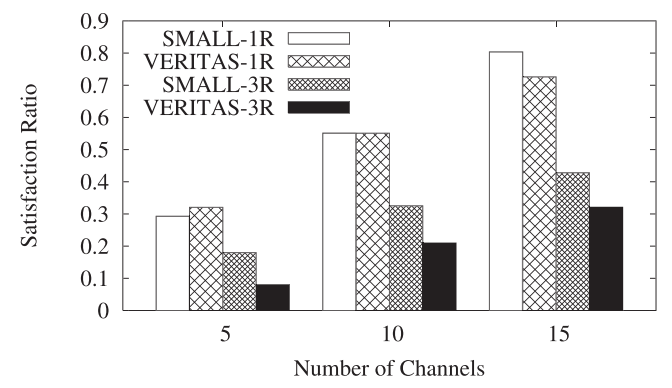

Fig. 4. Buyer satisfaction ratios of SMALL and VERITAS for auctioning 5,10 , and 15 channels among 200 buyers. All the buyers are equipped with one or three radios.

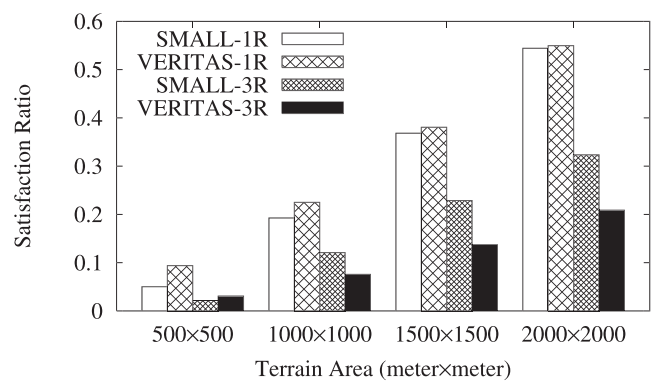

Fig. 5. Buyer satisfaction ratios of SMALL and VERITAS for auctioning 10 channels among 200 buyers. All the buyers are equipped with one or three radios.

satisfaction ratios to VERITAS in the single-radio case, and produces higher buyer satisfaction ratios than VERITAS in the multiradio case.

\subsection{Channel Utilization}

We present the simulation results on channel utilization in this section.

Fig. 6 shows channel utilizations of SMALL and VERITAS for auctioning 15 channels. Two sets of results are presented. In one set, each buyer only has a single radio. In the other set, each buyer is equipped with three radios. Again, SMALL's performance is worse than VERITAS when the number of (virtual) buyers is no larger than 120, but it outperforms VERITAS when the number of (virtual) buyers is larger than 120 .

Fig. 7 shows channel utilizations of SMALL and VERITAS for auctioning 5, 10, and 15 channels, among 200 buyers, when every buyer has a single radio and three radios, respectively. Again, the channel utilization of SMALL is higher than that of VERITAS, only except when every buyer has a single radio and there are five channels for sale.

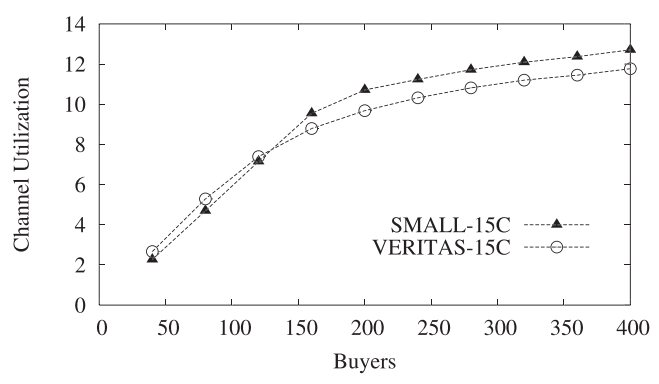

(a) Single radio

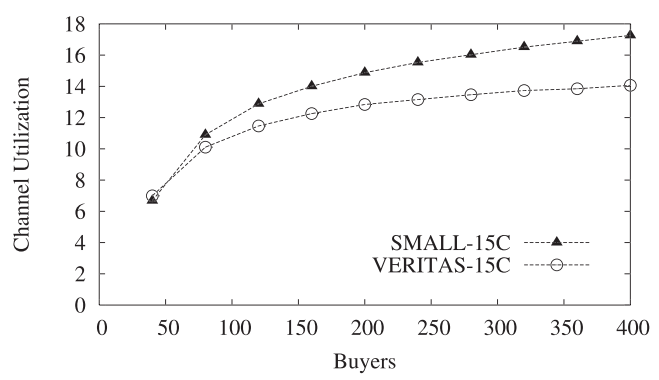

(b) 3 radios

Fig. 6. Channel utilizations of SMALL and VERITAS for auctioning 15 channels. 


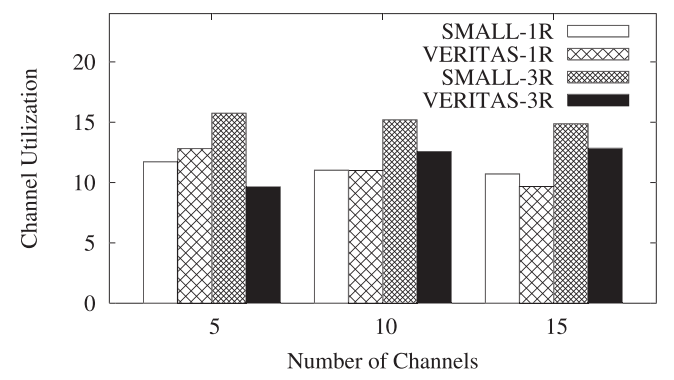

Fig. 7. Channel utilizations of SMALL and VERITAS for auctioning 5, 10, and 15 channels among 200 buyers. All the buyers are equipped with one or three radios.

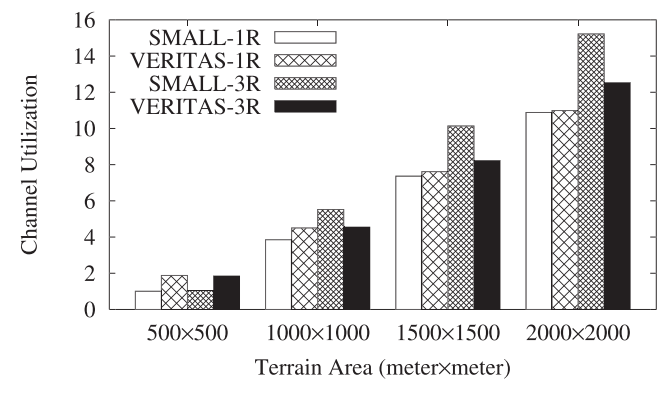

Fig. 8. Channel utilizations of SMALL and VERITAS for auctioning 10 channels among 200 buyers. All the buyers are equipped with one or three radios.

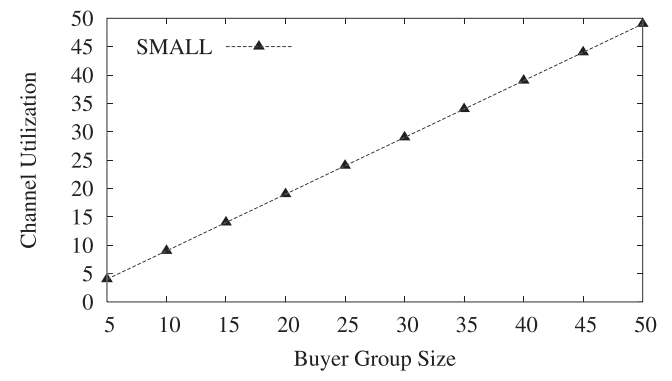

Fig. 9. Channel utilizations of SMALL for auctioning 10 channels as a function of buyer group size.

Fig. 8 shows the channel utilizations of SMALL and VERITAS, when there are 200 buyers and 10 channels for auctioning. The results for single-radio buyers and threeradio buyers are presented. Again, SMALL does not perform well when the terrain area is small $(500 \times 500$ meters). However, when the terrain area is relatively large $(1,000 \times 1,000,1,500 \times 1,500$, and $2,000 \times 2,000$ meters), SMALL achieves similar channel utilizations to VERITAS in the single-radio case, and reaches higher channel utilizations than VERITAS in the multiradio case.

Fig. 9 illustrates the channel utilization of SMALL for auctioning 10 channels as a function of buyer group size, when there are sufficient number of groups to buy the channels. Since one (virtual) buyer in each group must be sacrificed to guarantee truthfulness, the channel utilization is exactly the buyer group size minus one.

\subsection{Budget Efficiency}

We present the simulation results on budget efficiency in this section.

Since VERITAS does not consider seller's valuation/ reserve price on channels, we do not evaluate VERITAS in this set of simulations. Instead, we compare the overpayments of SMALL with another elegant channel auction

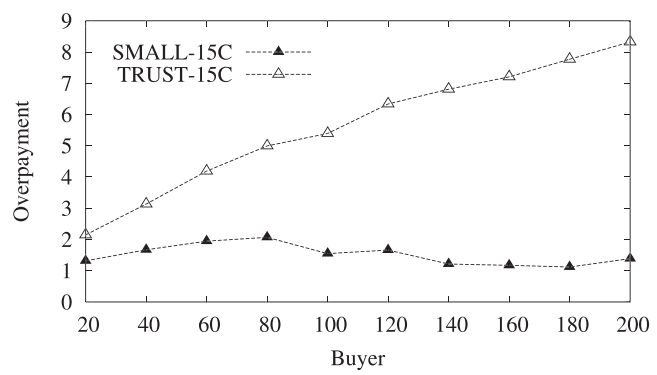

Fig. 10. Overpayments of SMALL and TRUST for auctioning 15 channels, when every buyer only has a single radio.

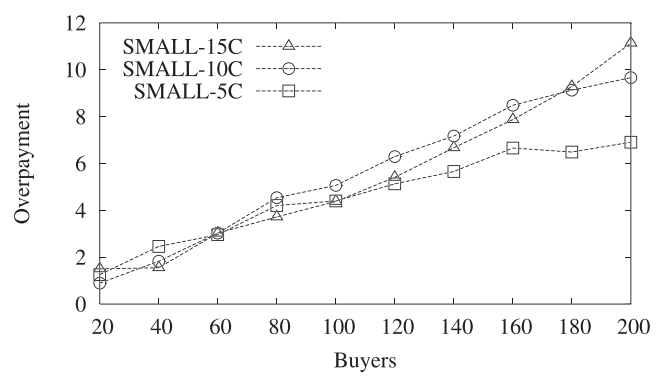

Fig. 11. Overpayments of SMALL for auctioning 5, 10, and 15 channels, when every buyer has three radios.

mechanism, namely TRUST, which is based on double auction. Since TRUST does not work in the scenario of multiple radios, we only evaluate SMALL's budget efficiency when nodes are equipped with multiple radios. Simulation results show that SMALL always provides smaller overpayment than TRUST, when each buyer has a single radio; and the overpayment induced by SMALL is also small, when buyers have multiple radios.

Fig. 10 shows the overpayments of SMALL and TRUST, when every buyer only has a single radio. The number of channels for auctioning is 15. From the figure, we can observe that SMALL always achieves much lower overpayment than TRUST.

Fig. 11 shows the overpayments of SMALL, when each buyer has three radios. The number of channels for auctioning are 5,10 , and 15 . We can see that the overpayments grow almost linearly with the number of the buyer, and the differences between the three lines are not much. However, the overpayment of SMALL is still small, when buyers has multiple radios.

We note that the amount of overpayment is dependant on the distributions of buyers' channel valuations and seller's reserve prices. However, the comparison results of using different distribution functions are identical.

\section{Related Works}

In this section, we first review related works on channel allocation that assume cooperation of participants, and then review the works with selfish participants.

\subsection{Cooperated Channel Allocation Works}

The channel allocation problem was first studied in cellular networks. We refer to [12] for a comprehensive survey.

A number of works were presented for wireless LANs (WLANs). For instance, Mishra et al. [18] utilized weighted graph coloring to address channel allocation for WLANs. 
Mishra et al. [19] used client-driven mechanisms to address the joint problem of channel allocation and load balancing in centrally managed WLANs.

Channel allocation problems are also studied in wireless mesh networks (WMNs). For example, Alicherry et al. [1], Raniwala et al. [23], and Kodialam and Nandagopal [13] considered channel allocation together with routing or scheduling in order to maximize network throughput. Some other works (e.g., [22]) focused on the channel allocation problem in rural mesh networks built with directional antennas.

The channel allocation problem is also studied in other wireless networks, such as ad hoc networks (e.g., [15]) and software defined radio networks (e.g., [11]).

\subsection{Channel Allocation Works with Selfish Participants}

The related works described in Section 6.1 require that all buyers in the network must be cooperative. Here, cooperative means that the buyers unconditionally obey a central control or behave strictly according to prescribed protocol. However, this assumption is not valid when the network consists of selfish nodes, whose goals are to maximize their payoff. With the existence of selfish buyers, assigning radios to channels becomes a game.

In an earlier work, Felegyhazi et al. [8] studied Nash Equilibria in a static multiradio multichannel allocation game. Later, $\mathrm{Wu}$ et al. [30] proposed a mechanism to make the multiradio multichannel allocation game converges to a much stronger equilibrium state, called strongly dominant strategy equilibrium, in which optimal system throughput is achieved.

The most closely related works are TRUST [36] and VERITAS [35], both of which are auction-based spectrum allocation mechanisms achieving strategy proofness. TRUST considers both buyers and sellers' incentives, and elegantly integrate double auction and radio spectrum allocation. In contrast, VERITAS focus on spectrum buyers and support multiple needs of the buyers. Recently, Athena [37] was proposed to prevent collusion in the spectrum auction.

Another important related work on channel allocation game is [10], in which the authors proposed a graph coloring game model and discussed the price of anarchy under various topology conditions such as different channel numbers and bargaining strategies.

In wireless networks, game theoretic approaches are also used to study media access problems. For example, MacKenzie and Wicker [16] studied the selfish behavior of buyers in Aloha networks. Later, Čagalj et al. [4] and Konorski [14] used game-theoretic approaches to investigate the media access problem of selfish buyers in CSMA/ CA networks. In cognitive radio networks, Nie and Comaniciu [20] proposed a game theoretic framework to analyze the behavior of cognitive radios for distributed adaptive spectrum allocation.

There are also other works on incentive compatibility in wireless networks. Examples include those works on packet routing and forwarding in ad hoc networks [2], [3], [6], [24], [26], [27], [32], [33], [34].

\section{CONCLUSIONS AND FUtURE WORK}

In this paper, we have modeled the radio spectrum allocation problem as a sealed-bid reserve auction, and proposed a strategy-proof radio spectrum allocation mechanism, call SMALL. We have implemented SMALL and evaluated its truthfulness and performance. Compared with existing work VERITAS and TRUST, SMALL achieves better performance in median to large scale spectrum auctions, in terms of buyer satisfaction ratio, spectrum utilization, and budget efficiency. As for future work, we are interested in designing similar simple mechanisms that can prevent collusion among multiple spectrum buyers.

\section{ACKNOWLEDGMENTS}

Part of the results in this paper appeared at the IEEE INFOCOM 2011 Mini-Conference. This work was supported in part by US National Science Foundation (NSF) grant 06-27074, in part by US Army Research Office grant W911NF-05-1-0246, in part by China NSF grant 61170236 and 61133006, and in part by Key Project of Educational Science Research of Shanghai grant A1120. The opinions, findings, conclusions, and recommendations expressed in this paper are those of the authors and do not necessarily reflect the views of the funding agencies or the government.

\section{References}

[1] M. Alicherry, R. Bhatia, and L. Li, “Joint Channel Assignment and Routing for Throughput Optimization in Multi-Radio Wireless Mesh Networks," Proc. ACM MobiCom, Sept. 2005.

[2] L. Anderegg and S. Eidenbenz, "Ad Hoc-VCG: A Truthful and Cost-Efficient Routing Protocol for Mobile Ad Hoc Networks with Selfish Agents," Proc. ACM MobiCom, Sept. 2003.

[3] N. Ben Salem, L. Buttyan, J.P. Hubaux, and M. Jakobsson, "A Charging and Rewarding Scheme for Packet Forwarding in MultiHop Cellular Networks," Proc. ACM MobiHoc, Jun. 2003.

[4] M. Cagalj, S. Ganeriwal, I. Aad, and J.-P. Hubaux, "On Selfish Behavior in CSMA/CA Networks," Proc. IEEE INFOCOM, Apr. 2005.

[5] D.C. Cox and D.O. Reudink, "Dynamic Channel Assignment in High Capacity Mobile Communication System," Bell System Technical J., vol. 50, no. 6, pp. 1833-1857, 1971.

[6] S. Eidenbenz, G. Resta, and P. Santi, "COMMIT: A Sender-Centric Truthful and Energy-Efficient Routing Protocol for Ad Hoc Networks with Selfish Nodes," Proc. 19th Int'l Parallel and Distributed Processing Symp. (IPDPS), Apr. 2005.

[7] Federal Communication Commission (FCC), http://www.fcc.gov, 2012.

[8] M. Félegyházi, M. Čagalj, S.S. Bidokhti, and J.-P. Hubaux, "NonCooperative Multi-Radio Channel Allocation in Wireless Networks," Proc. IEEE INFOCOM, May 2007.

[9] D. Fudenberg and J. Tirole, Game Theory. MIT Press, 1991.

[10] M.M. Halldórsson, J.Y. Halpern, L.E. Li, and V.S. Mirrokni, "On Spectrum Sharing Games," Proc. 23rd Ann. ACM SIGACT-SIGOPS Symp. Principles of Distributed Computing (PODC), July 2004.

[11] Y.T. Hou, Y.Shi, and H.D. Sherali, "Optimal Spectrum Sharing for Multi-Hop Software Defined Radio Networks," Proc. IEEE INFOCOM, May 2007.

[12] I. Katzela and M. Naghshineh, "Channel Assignment Schemes for Cellular Mobile Telecommunications: A Comprehensive Survey," IEEE Personal Comm., vol. 3, no. 3, pp. 10-31, June 1996.

[13] M. Kodialam and T. Nandagopal, "Characterizing the Capacity Region in Multi-Radio Multi-Channel Wireless Mesh Networks," Proc. ACM MobiCom, Sept. 2005.

[14] J. Konorski, "Multiple Access in Ad-Hoc Wireless LANs with Noncooperative Stations," Proc. IFIP Second Int'l IFIP-TC6 Networking Conf. Networking Technologies, Services, and Protocols; Performance of Computer and Comm. Networks; and Mobile and Wireless Comm. (Networking '02), May 2002. 
[15] P. Kyasanur and N. Vaidya, "A Routing Protocol for Utilizing Multiple Channels in Multi-Hop Wireless Networks with a Single Transceiver," Proc. Second Int'l Conf. Quality of Service in Heterogeneous Wired/Wireless Networks (QShine), Aug. 2005.

[16] A.B. MacKenzie and S.B. Wicker, "Stability of Multipacket Slotted Aloha with Selfish Users and Perfect Information," Proc. IEEE INFOCOM, Apr. 2003.

[17] A. Mas-Colell, M.D. Whinston, and J.R. Green, Microeconomic Theory. Oxford, 1995.

[18] A. Mishra, S. Banerjee, and W. Arbaugh, "Weighted Coloring Based Channel Assignment for WLANs," ACM SIGMOBILE Mobile Computing and Comm. Rev., vol. 9, no. 3, pp. 19-31, 2005.

[19] A. Mishra, V. Brik, S. Banerjee, A. Srinivasan, and W. Arbaugh, "A Client-Driven Approach for Channel Management in Wireless LAN," Proc. IEEE INFOCOM, Apr. 2006.

[20] N. Nie and C. Comaniciu, "Adaptive Channel Allocation Spectrum Etiquette for Cognitive Radio Networks," Proc. IEEE First Int'l Symp. New Frontiers in Dynamic Spectrum Access Networks (DySPAN), Nov. 2005.

[21] M.J. Osborne and A. Rubenstein, A Course in Game Theory. MIT, 1994.

[22] B. Raman, "Channel Allocation in 802.11-Based Mesh Networks," Proc. IEEE INFOCOM, Apr. 2006.

[23] A. Raniwala, K. Gopalan, and T. cker Chiueh, "Centralized Channel Assignment and Routing Algorithms for Multi-Channel Wireless Mesh Networks," ACM SIGMOBILE Mobile Computing and Comm. Rev., vol. 8, no. 2, pp. 50-65, 2004.

[24] V. Srinivasan, P. Nuggehalli, C.-F. Chiasserini, and R. Rao, "Cooperation in Wireless Ad Hoc Networks," Proc. IEEE INFOCOM, Apr. 2003.

[25] H. Varian, "Economic Mechanism Design for Computerized Agents," Proc. USENIX Workshop Electronic Commerce, 1995.

[26] W. Wang, S. Eidenbez, Y. Wang, and X.-Y. Li, "Ours-Optimal Unicast Routing Systems in Non-Cooperative Wireless Networks," Proc. ACM MobiCom, Sept. 2006.

[27] W. Wang, X.-Y. Li, and Y. Wang, "Truthful Multicast in Selfish Wireless Networks," Proc. ACM MobiCom, Sept. 2004.

[28] D.J.A. Welsh and M.B. Powell, "An Upper Bound for the Chromatic Number of a Graph and Its Application to Timetabling Problems," The Computer J., vol. 10, no. 1, pp. 85-86, 1967.

[29] D.B. West, Introduction to Graph Theory, second ed. Prentice Hall, 1996.

[30] F. Wu, S. Zhong, and C. Qiao, "Globally Optimal Channel Assignment for Non-Cooperative Wireless Networks," Proc. IEEE INFOCOM, Apr. 2008.

[31] W. Yue, "Analytical Methods to Calculate the Performance of a Cellular Mobile Radio Communication System with Hybrid Channel Assignment," IEEE Trans. Vehicular Technology, vol. 40, no. 2, pp. 453-460, May 1991.

[32] S. Zhong, L.E. Li, Y.G. Liu, and Y.R. Yang, "On Designing Incentive-Compatible Routing and Forwarding Protocols in Wireless Ad-Hoc Networks: An Integrated Approach Using Game Theoretical and Cryptographic Techniques," Proc. ACM MobiCom, Sept. 2005.

[33] S. Zhong, J. Chen, and Y.R. Yang, "Sprite: A Simple, Cheat-Proof, Credit-Based System for Mobile Ad-Hoc Networks," Proc. IEEE INFOCOM, Apr. 2003.

[34] S. Zhong and F. Wu, "On Designing Collusion-Resistant Routing Schemes for Non-Cooperative Wireless Ad Hoc Networks," Proc. ACM MobiCom, Sept. 2007.

[35] X. Zhou, S. Gandhi, S. Suri, and H. Zheng, "eBay in the Sky: Strategy-Proof Wireless Spectrum Auctions," Proc. ACM MobiCom, Sept. 2008.

[36] X. Zhou and H. Zheng, "TRUST: A General Framework for Truthful Double Spectrum Auctions," Proc. IEEE INFOCOM, Apr. 2009

[37] X. Zhou and H. Zheng, "Breaking Bidder Collusion in Large-Scale Spectrum Auctions," Proc. ACM MobiHoc, June 2010.

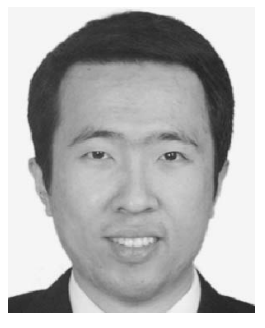

Fan $\mathbf{W u}$ received the $\mathrm{BS}$ degree in computer science from Nanjing University in 2004 and the $\mathrm{PhD}$ degree in computer science and engineering from the State University of New York at Buffalo in 2009. He is an associate professor in the Department of Computer Science and Engineering at Shanghai Jiao Tong University, P.R. China. He has visited the University of Illinois at Urbana-Champaign (UIUC) as a postdoctoral research associate. His research interests include wireless networking, economic incentives for cooperation, and peer-to-peer computing. He is a member of the IEEE and the IEEE Computer Society. For more information, please visit http:// www.cs.sjtu.edu.cn/ fwu.

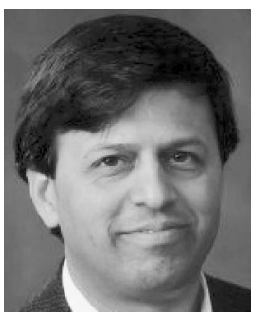

Nitin Vaidya received the $\mathrm{PhD}$ degree from the University of Massachusetts at Amherst. $\mathrm{He}$ is a professor of Electrical and Computer Engineering at the University of Illinois at Urbana-Champaign (UIUC), where he previously also served as the director of the Illinois Center for Wireless Systems (ICWS). $\mathrm{He}$ has held visiting positions at Thomson Research, Paris, the Indian Institute of Technology Bombay, Microsoft Research, and Sun Microsystems, as well as a faculty position at the Texas A\&M University. He coauthored papers that received awards at the 1998 ACM MobiCom, 2007 ACM MobiHoc, 2003 Personal Wireless Communications (PWC), 2010 ICDCN, and 2010 WWIC conferences. $\mathrm{He}$ is a recipient of a CAREER award from the US National Science Foundation. He served as editor-in-chief for the IEEE Transactions on Mobile Computing, editor-in-chief for the ACM SIGMOBILE publication $M C 2 R$, and general chair for the 2010 joint ACM MobiCom-MobiHoc conferences. He is a fellow of the IEEE. For more information, please visit http://users.crhc.illinois.edu/nhv.

For more information on this or any other computing topic, please visit our Digital Library at www.computer.org/publications/dlib. 